\title{
L-3-(4-吡啶基)-丙氨酸钝化钙钛矿太阳电池界面缺陷
}

\author{
刘雯雯 ${ }^{1}$, 胡志蕾 ${ }^{1}$, 王 立 ${ }^{1}$, 曹梦莎 ${ }^{1}$, 张 晶 $^{1}$, 张 婧 ${ }^{1}$, \\ 张 帅 ${ }^{1}$ ，袁宁一 1 ，丁建宁 ${ }^{2}$
}

(1. 常州大学 江苏省光伏科学与工程协同创新中心 材料科学与工程学院, 常州 $213164 ; 2$. 江苏大学微纳科学与 技术中心，镇江 212013)

摘 要: 近年来钙钛矿材料因其优异的光电性能而成为光伏领域的研究热点, 但调控钻钛矿太阳电池内界面缺陷仍 是亟需解决的关键问题之一。本研究在溶液两步法制备钙钛矿光吸收层的过程中引入有机小分子添加剂 $(L-3-(4$ 吡 啶基)-丙氨酸(L-3-(4-pyridyl)-alanine, (PLA))。测试结果显示引入 PLA 可提高器件的各光电性能参数, 含 PLA 器件 的最优能量转换效率为 $21.53 \%$, 而参照器件为 $20.10 \%$ 。进一步研究表明引入 PLA 可延长苂光寿命, 降低器件的陷 阱态密度 (从 $5.59 \times 10^{16} \mathrm{~cm}^{-3}$ 降至 $3.40 \times 10^{16} \mathrm{~cm}^{-3}$ ), 促进界面电荷抽取, 抑制载流子复合。器件性能的提升是由于 PLA 促进 $\mathrm{PbI}_{2}$ 在钙钛矿薄膜晶界处富集及 PLA 在界面处针定起到了针化缺陷的作用。本研究可以为进一步调控钙钛矿 太阳电池的缺陷提供借鉴。

关＼cjkstart键 词：钙钛矿太阳电池；多官能团添加剂；陷阱态密度；载流子复合

中图分类号: 0649 文献标志码: A

\section{Passiviation of $L$-3-(4-Pyridyl)-alanine on Interfacial Defects of Perovskite Solar Cell}

\author{
LIU Wenwen ${ }^{1}$, HU Zhilei ${ }^{1}$, WANG Li $^{1}$, CAO Mengsha ${ }^{1}$, ZHANG Jing ${ }^{1}$, ZHANG Jing ${ }^{1}$, \\ ZHANG Shuai $^{1}$, YUAN Ningyi ${ }^{1}$, DING Jianning ${ }^{2}$
}

(1. School of Materials Science and Engineering, Jiangsu Photovoltaic Science and Engineering Collaborative Innovation Center, Changzhou university, Changzhou 213164, China; 2. Micro Center for Science and Technology, Jiangsu University, Zhenjiang 212013, China)

\begin{abstract}
In recent years, perovskite materials become a research hotspot in the field of solar cells due to their excellent photovoltaic properties, but control of their interface defects still remains one of the key problems to be solved. In this study, an organic small molecule additive (L-3-(4-pyridyl)-alanine (PLA)) was introduced in the preparation of perovskite photoabsorption layer by two-step solution method. The characterizations showed that the introduction of PLA could comprehensively improve photoelectric performance of the device, with optimal energy conversion efficiency of $21.53 \%$, in contrast to that of the reference device $(20.10 \%)$. Further studies showed that PLA
\end{abstract}

收稿日期: 2020-08-27; 收到修改稿日期：2020-10-22；网络出版日期：2020-11-05

基金项目: 国家自然科学基金(51602031，51603021); 江苏省“青蓝工程”优秀青年骨干教师(2019); 江苏省高等学校自然科 学研究重大项目(19KJA430014); 江苏省研究生科研与实践创新计划(SJCX20_0975)

National Natural Science Foundation of China (51602031, 51603021); The Qinlan Project (2019); Natural Science Foundation of the Jiangsu Higher Education Institutions (19KJA430014); Postgraduate Research \& Practice Innovation Program of Jangsu Province (SJCX20_0975)

作者简介: 刘雯雯(1997-), 女, 硕士研究生. E-mail: 2356143925@qq.com LIU Wenwen(1997-), female, Master candidate. E-mail: 2356143925@qq.com

通信作者：张 帅，副教授. E-mail: shuaizhang@cczu.edu.cn; 丁建宁，教授. E-mail: dingjn@cczu.edu.cn ZHANG Shuai, associate professor. E-mail: shuaizhang@cczu.edu.cn; DING Jianning, professor. E-mail: dingjn@ cczu.edu.cn 
could reduce the trap state density of the device from $5.59 \times 10^{16} \mathrm{~cm}^{-3}$ to $3.40 \times 10^{16} \mathrm{~cm}^{-3}$, promote the interface charge extraction, and decrease the carrier recombination. The above improvements can be attributed to the PLA induced $\mathrm{PbI}_{2}$ enrichment at grain boundaries and PLA anchoring at defects, which play an important roles in passivate defects. This study can provide guideline for further regulating the defects of perovskite solar cells.

Key words: perovskite solar cell; additive with multifunctional groups; trap state density; carrier recombination

有机铅卤化物钙钛矿太阳电池(Perovskite Solar Cell, PSC)的光电转换效率(Photon-to-electron conversion efficiency, PCE) 由 2009 年报道的 $3.8 \%{ }^{[1]}$ 上升至目前 的最高认证效率 $25.5 \%{ }^{[2]}$, 近年来一直是光伏领域 的研究热点。然而, 器件界面缺陷仍是亟需解决的 关键问题之一, 例如电子传输层(Electron transport layer, $\mathrm{ETL}) \mathrm{TiO}_{2}$ 表面的带电缺陷、钙钛矿薄膜晶界 处未配位有机阳离子和卤素空位导致的缺陷等。 PSC 的界面缺陷会造成载流子复合和离子迁移, 导 致器件光电性能下降和迟滞。因此, 钝化界面缺陷 成为提升 PSC 性能的重要方法 ${ }^{[3-6]}$ 。

在 PSC 内部引入添加剂是修饰器件界面缺陷的 重要方法之一。研究表明, 添加剂可通过调控钻钛 矿薄膜的结晶过程来改善薄膜质量、抑制缺陷 ${ }^{[7-8]}$, 或与钙铁矿材料特定缺陷位点相互作用来钝化缺陷, 提升性能 ${ }^{[9-10]}$ 。在实现上述功能的过程中, 添加剂分 子的特定官能团起到了重要的作用, 例如羧基 ${ }^{[11]}$ 、 氨基 ${ }^{[12]}$ 、羰基 ${ }^{[13]}$ 、吡啶 ${ }^{[14]}$ 、卤素原子 ${ }^{[7]}$ 等。然而，现 有报道的添加剂基本只含有一种官能团, 少数含有 两种 ${ }^{[11]}$ 。含有多种官能团的添加剂分子可能同时对 钙铁矿材料的多种缺陷具有修饰作用, 因此探索含 有多种官能团的添加剂分子对钝化钙钛矿材料缺陷 和提升器件性能具有重要的意义。

钙钛矿薄膜晶界处富集 $\mathrm{PbI}_{2}$ 和在 $\mathrm{TiO}_{2}$ 表面和钙 钛矿晶界处针定 PLA 分子可以提升器件光电性能。 其中, 钙钛矿薄膜晶界处富集 $\mathrm{PbI}_{2}$ 可延长载流子寿 命、降低卤素空位浓度 ${ }^{[15]}$ 。此外，根据文献[8]报道， 羧基和氨基可通过静电相互作用中和 $\mathrm{TiO}_{2}$ 的带电 缺陷，而吡啶中的氮原子可以和钙钛矿未配位的有 机阳离子形成氢键 ${ }^{[14]}$, 因此 PLA 添加剂可针定上述 缺陷位点, 通过给出电子, 消除电子陷阱, 抑制载 流子复合, 促进其输运, 从而提升器件的 PCE, 抑 制迟滞。因此, 本研究采用溶液两步法制备钙钛矿 薄膜, 针对 $\mathrm{TiO}_{2}$ 表面带电缺陷和钙钛矿薄膜晶界处 存在的未配位有机阳离子及卤素空位, 选择同时含 有羧基、氨基和吡啶的添加剂( $L-3$-(4-吡啶基)-丙氨 酸(PLA))进行修饰。获得的样品通过 $X$ 射线衍射 (XRD)、扫描电子显微镜(SEM)、光致发光、空间电 荷限制电流法(Space-charge limited current, SCLC)、
交流阻抗谱(Electrochemical impedence spectroscopy, EIS)等手段来验证 PLA 的修饰效果, 探讨 PLA 提升 PSC 光电性能的机理。

\section{1 实验方法}

\section{1 实验试剂}

溴化铅 $\left(\mathrm{PbBr}_{2}, 99.999 \%\right)$ 、碘化铯(CsI, 99.9\%)、 甲脒氢碘酸盐( $\mathrm{FAI},>99.5 \%$ )、甲胺氢溴酸盐( $\mathrm{MABr}$, $>99.5 \%$ )、甲胺盐酸盐 $(\mathrm{MACl},>99.5 \%) 、 2,2^{\prime}, 7,7^{\prime}$-四 [ $N, N$-二 (4- 甲氧基苯基) 氨基] $-9,9^{\prime}$ - 螺二芴 (Spiro-OMeTAD, >99.5\%)、[6, 6]-苯基-C61-丁酸异 甲酯(PCBM，>99\%)等试剂购自西安宝莱特光电科 技有限公司。碘化铅 $\left(\mathrm{PbI}_{2}, 99.99 \%\right)$ 和乙腈( $\left.>99.5 \%\right)$ 购于东京化学工业有限公司。添加剂 PLA (95\%)购 于 Key Organics 公司。 $N, N$-二甲基甲酰胺(DMF, $99.9 \%)$ 、二甲基亚砜( DMSO, $99.9 \%)$ 、三 $(2-(1 \mathrm{H}-$ 吡 唑-1-基)-4-叔丁基吡啶)-钴( II )三(双 (三氟甲基磺酰) 亚胺))(FK209Co( II ), 99.9\%)、双三氟甲烷磺酰亚胺 锂(Li-TFSI, $>99.5 \%$ )购自 SIGMA-ALDRICH 试剂公 司。无水乙醇(AR)、丙酩(AR)购自国药集团化学试剂 有限公司。四氯化钛 $\left(\mathrm{TiCl}_{4}, 99.0 \%\right) 、 4$-叔丁基吡啶 (TBP, $>96 \%)$, 氯苯 $(\mathrm{CB}, \mathrm{AR})$ 购自阿拉丁试剂有限公司。

\section{2 钙钛矿太阳电池的制备}

\subsection{1 $\mathrm{TiO}_{2}$ 电子传输层 $(\mathrm{ETL})$ 的制备}

将 FTO 玻璃依次用去离子水和乙醇超声清洗 $20 \mathrm{~min}$, 烘干后浸入 $\mathrm{TiCl}_{4}$ 水溶液 $\left(0.225 \mathrm{~mol} \cdot \mathrm{L}^{-1}\right)$ 中, 在 $70{ }^{\circ} \mathrm{C}$ 烘箱中反应 $80 \mathrm{~min}$; 反应完成后用去离子 水清洗并烘干, 得到 $\mathrm{FTO} / \mathrm{TiO}_{2}$ 。

\subsection{2 钙钛矿光吸收层的制备}

实验前将 $\mathrm{FTO} / \mathrm{TiO}_{2}$ 衬底紫外臭氧处理 $20 \mathrm{~min}$ 。 在 $\mathrm{FTO} / \mathrm{TiO}_{2}$ 祄底上旋涂 $(1500 \mathrm{r} / \mathrm{min}, 30 \mathrm{~s}) 25 \mu \mathrm{L}$ $1.5 \mathrm{~mol} \cdot \mathrm{L}^{-1} \mathrm{PbI}_{2}$ 溶液 $\left(V_{\mathrm{DMF}}: V_{\mathrm{DMSO}}=9: 1,70{ }^{\circ} \mathrm{C}\right.$ 下退 火 $10 \mathrm{~s}$; 然后在 $\mathrm{FTO} / \mathrm{TiO}_{2} / \mathrm{PbI}_{2}$ 衬底上旋涂 $(2000 \mathrm{r} / \mathrm{min}$, $30 \mathrm{~s}) 18 \mu \mathrm{L}$ 含 $90 \mathrm{mg} \cdot \mathrm{mL}^{-1} \mathrm{FAI} 、 6.39 \mathrm{mg} \cdot \mathrm{mL}^{-1} \mathrm{MABr}$ 和 $9 \mathrm{mg} \cdot \mathrm{mL}^{-1} \mathrm{MACl}$ 的异丙醇溶液, 在大气环境中 (湿度 30\%) $150{ }^{\circ} \mathrm{C}$ 下退火 $15 \mathrm{~min}$, 获得的 $\mathrm{FTO} / \mathrm{TiO}_{2} /$ 钙钛矿为参照样品。在旋涂有机盐溶液前旋涂 $30 \mu \mathrm{L}$ 
$0.1 \mathrm{mg} \cdot \mathrm{mL}^{-1} \mathrm{PLA}$ 甲醇溶液 $(4500 \mathrm{r} / \mathrm{min}, 30 \mathrm{~s}$ ), 随后 $70{ }^{\circ} \mathrm{C}$ 下退火 $10 \mathrm{~s}$, 其他步骤相同, 可获得 PLA 修 饰的 $\mathrm{FTO} / \mathrm{TiO}_{2} /$ 钙钛矿。此外, 采用相同的方法在不 含 $\mathrm{TiO}_{2}$ 层的玻璃祄底上制备钻钛矿吸收层, 用于光 致发光表征。

\subsection{3 空穴传输层(Hole transport layer, HTL)及电 极制备}

将 $72.8 \mathrm{mg}$ spiro-MeOTAD 溶于 $1 \mathrm{~mL} \mathrm{CB}$ 中, 添加 $32.4 \mu \mathrm{L}$ TBP、 $17.4 \mu \mathrm{L} 520 \mathrm{mg} \cdot \mathrm{mL}^{-1} \mathrm{Li}$-TFSI 的乙腈溶 液、 $29 \mu \mathrm{L} 300 \mathrm{mg} \cdot \mathrm{mL}^{-1} \mathrm{KF} 209 \mathrm{Co}(\mathrm{II})$ 的乙腈溶液, 得 到 HTL 溶液。然后在 $\mathrm{FTO} / \mathrm{TiO}_{2} /$ 钻钛矿祄底上旋涂 $(4500 \mathrm{r} / \mathrm{min}, 30 \mathrm{~s}) 20 \mu \mathrm{L}$ 的 $\mathrm{HTL}$ 溶液, 得到 $\mathrm{FTO} / \mathrm{TiO}_{2} /$ 钙钛矿/Spiro-MeOTAD。此外, 还在 FTO/ $\mathrm{TiO}_{2} /$ 钙钛 矿祄底上旋涂 $(5000 \mathrm{r} / \mathrm{min}, 5 \mathrm{~s} ; 3000 \mathrm{r} / \mathrm{min}, 85 \mathrm{~s}) 20 \mu \mathrm{L}$ $20 \mathrm{mg} \cdot \mathrm{mL}^{-1} \mathrm{PCBM}$ 的苯甲醚溶液, 在 $75{ }^{\circ} \mathrm{C}$ 下退火 $5 \mathrm{~min}$, 得到 $\mathrm{FTO} / \mathrm{TiO}_{2} /$ 钙钣矿/ $\mathrm{PCBM}$ 。随后将两种样品置 于高真空电阻蒸发仪中蒸镀厚度为 $80 \mathrm{~nm}$ 的金电极, 获得 PSC 器件( $\mathrm{FTO} / \mathrm{TiO}_{2} /$ 钙钛矿/Spiro-MeOTAD/Au) 和 纯电子注入器件 $\left(\mathrm{FTO} / \mathrm{TiO}_{2} /\right.$ 钙钛矿/ $\left.\mathrm{PCBM} / \mathrm{Au}\right)$, 后 者用于 SCLC 表征, 器件的有效面积为 $0.07 \mathrm{~cm}^{2}$ 。 以上步骤如无特殊说明均在手套箱中完成, 图 1 为 PLA 分子结构式和 PSC 器件的结构示意图。

\section{3 仪器测试及表征}

本研究采用 SEM (Zeiss SUPRA55)、XRD (Rigaku $\mathrm{D} / \mathrm{Max} 2500 \mathrm{PC}$ )、紫外-可见分光光度计(岛津 UV3600) 和傅里叶变换衰减全反射红外光谱(ATR-FT-IR, Nicolet IS10)分别表征钙钣矿薄膜的形貌、光学性能和分 子结构; 使用光致发光测试系统(Horiba DeltaFlex PL) 记录钙铁矿薄膜的稳态荧光 (Photouminescence, PL) 和时间分辨荧光 (Time resolved fluorescence photoluminescence, TRPL), 表征钙钛矿薄膜的载流 子寿命; 利用电化学工作站(Zahner IM6ex)测试器件 的 EIS 图谱; 利用外量子效率(External quantum efficiency, EQE)测试系统验证器件的 $J-V$ 测试结果。 器件的光电性能均使用太阳光模拟器 (94023A SOLAR SIM S/N: 387)在 AM $1.5 \mathrm{G}$ 条件下进行测试。 (a)<smiles>N[C@@H](Cc1ccncc1)C(=O)O</smiles>

(b)

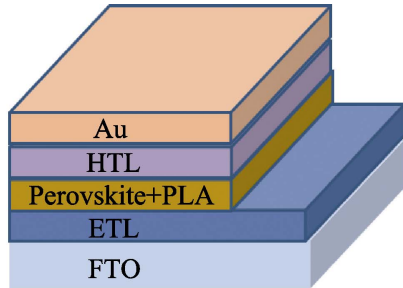

图 1 (a)PLA 的分子结构式和(b)PLA 修饰的 PSC 器件结构 示意图

Fig. 1 (a) Molecular structure of PLA and (b) schematic diagram of PSC device with PLA

\section{2 结果与讨论}

\subsection{PLA 修饰对钙钛矿薄膜的影响}

本研究的钙钛矿薄膜采用溶液两步法制备, 首 先添加 PLA 分子到 $\mathrm{PbI}_{2}$ 薄膜中。图 S1 为 PLA 修饰 及参照 $\mathrm{FTO} / \mathrm{TiO}_{2} / \mathrm{PbI}_{2}$ 薄膜的表面和截面 $\mathrm{SEM}$ 照片, 与参照样品相比, 引入 PLA 分子未改变 $\mathrm{PbI}_{2}$ 薄膜的 表面形貌(图 S1(a,b)), 但含 PLA 的样品截面有微弱 的分层现象，这可能是 PLA 甲醇溶液冲洗造成的。 该分层使 $\mathrm{PbI}_{2}$ 薄膜更为疏松, 有利于后续有机盐溶 液的渗入并与 $\mathrm{PbI}_{2}$ 充分反应, 形成高质量的钙钛矿 薄膜。

图 2(a,b)为 $\mathrm{FTO} / \mathrm{TiO}_{2} /$ 钙针矿薄膜的表面 SEM 照片, 薄膜表面光滑平整, 为致密多晶结构。PLA 修饰钙钛矿薄膜的多数晶界表现为高亮区域(图 2(b)), 而参照钙钛矿薄膜则不明显。高亮区域表明晶界处 有更多的 $\mathrm{PbI}_{2}$ 富集 ${ }^{[15]}$, 这是 $\mathrm{PbI}_{2}$ 晶粒表面铅离子和 PLA 吡啶基团中的氮原子形成非共价键而造成 的 ${ }^{[14]}$ 。根据相关报道 ${ }^{[15-16]}$, 晶界处富集的 $\mathrm{PbI}_{2}$ 可延 长载流子寿命并降低卤素空位浓度, 能够钝化钙钛 矿薄膜晶界处的缺陷。晶粒尺寸分布图(图 2(e, f)) 显示 PLA 修饰薄膜的平均晶粒尺寸约为 $0.9 \mu \mathrm{m}$, 略 小于参照薄膜 $(1.0 \mu \mathrm{m})$, 但尺寸分布更窄。钙钛矿 薄膜的截面 SEM 照片(图 2(c, d))显示两种薄膜的
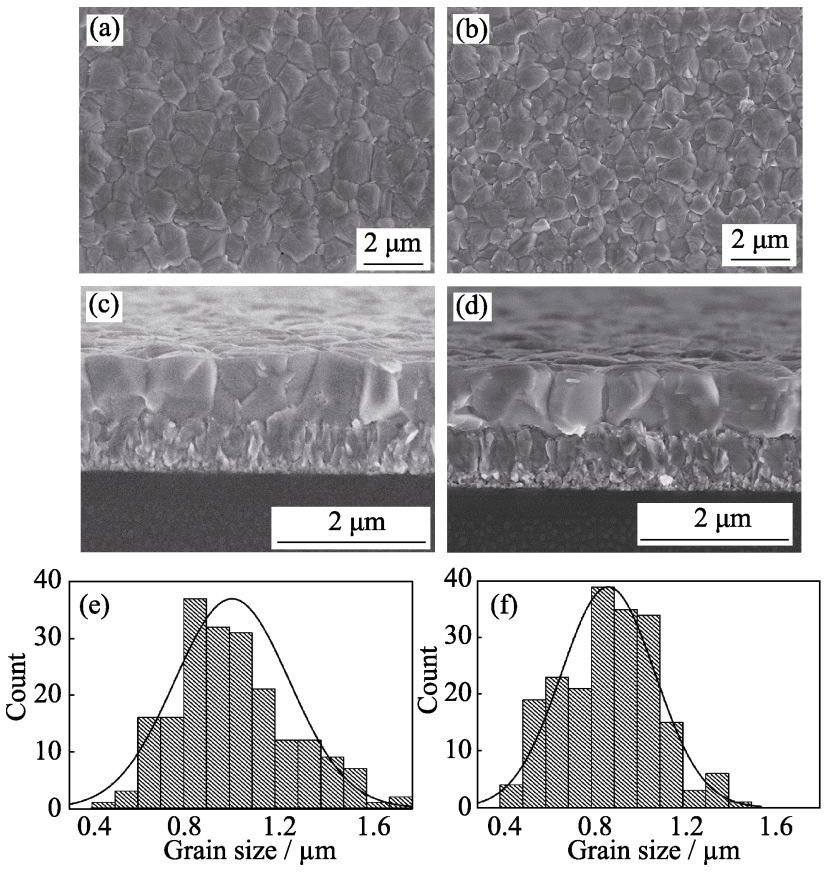

图 $2(\mathrm{a}, \mathrm{c}, \mathrm{e})$ 参照及 $(\mathrm{b}, \mathrm{d}, \mathrm{f}) \mathrm{PLA}$ 修饰 $\mathrm{FTO} / \mathrm{TiO}_{2} /$ 钻钛矿薄膜 的(a,b)表面、 $(c, d)$ 截面 SEM 照片和(e,f)晶粒尺寸分布图 Fig. 2 (a,b) Surface and (c,d) cross sectional SEM images, $(\mathrm{e}, \mathrm{f})$ grain size distributions of $\mathrm{FTO} / \mathrm{TiO}_{2} /$ perovskite films (a, c, e) without and (b, d, f) with PLA 
晶粒均可贯穿整个钻钛矿层, 且垂直致密, 有利于 载流子输运。两种钙钛矿薄膜的厚度接近, 约为 $740 \mathrm{~nm}$ 。由于钙钛矿材料的光吸收系数高 ${ }^{[17]}$, 载流 子扩散长度可达 $1 \mu \mathrm{m}^{[18]}$, 该薄膜厚度可保证薄膜 兼具良好的光吸收 ${ }^{[19]}$ 和载流子输运性能。

为确认钙钛矿薄膜中存在 PLA 分子, 对薄膜进 行 ATR-FT-IR 表征。对比参照钙钛矿薄膜, PLA 修饰 薄膜在 $1400 \mathrm{~cm}^{-1}$ 附近出现了新的吸收峰(图 3(a, b)), 归属于 PLA 分子中的 $v_{\mathrm{s}}\left(\mathrm{COO}^{-}\right)$, 这表明 PLA 分子确 实被引入钙针矿层。此外, 位于 3260 和 $3400 \mathrm{~cm}^{-1}$ 附 近的吸收峰归属于 $v\left(\mathrm{~N}-\mathrm{H}_{3}{ }^{+}\right)$, 位于 $1700 \mathrm{~cm}^{-1}$ 附近 的吸收峰归属于 $v(\mathrm{C}=\mathrm{O})^{[20]}$ 。对两种钙钛矿薄膜进行 紫外-可见吸收光谱表征如图 3(c) 所示, 两者的光学 带隙接近, 均为 $1.61 \mathrm{eV}$, PLA 修饰薄膜较参照薄膜 在可见光区域内光吸收性能更佳。对薄膜进行 XRD 表征发现两者均在 $2 \theta=14.0^{\circ} 、 28.3^{\circ} 、 31.6^{\circ}$ 处有明显 的衍射峰(图 3(d)), 分别对应立方相钙钛矿的(100)、

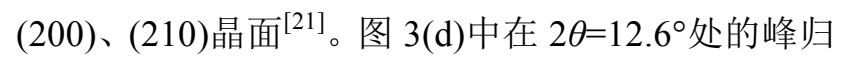
属于 $\mathrm{PbI}_{2}(001)$ 晶面 ${ }^{[22]}$, 这与报道的两步法制备钙 钛矿薄膜的 XRD 结果一致。此外, PLA 修饰钙钛矿 薄膜的 $\mathrm{PbI}_{2}$ 衍射峰强度要弱于参照薄膜, 而钙钛矿 (100)晶面衍射峰强度更强。这可能是由于 PLA 溶液 冲洗导致的 $\mathrm{PbI}_{2}$ 薄膜分层, 有利于 $\mathrm{PbI}_{2}$ 和有机盐的 反应, 促进了钻钛矿晶体的生长。
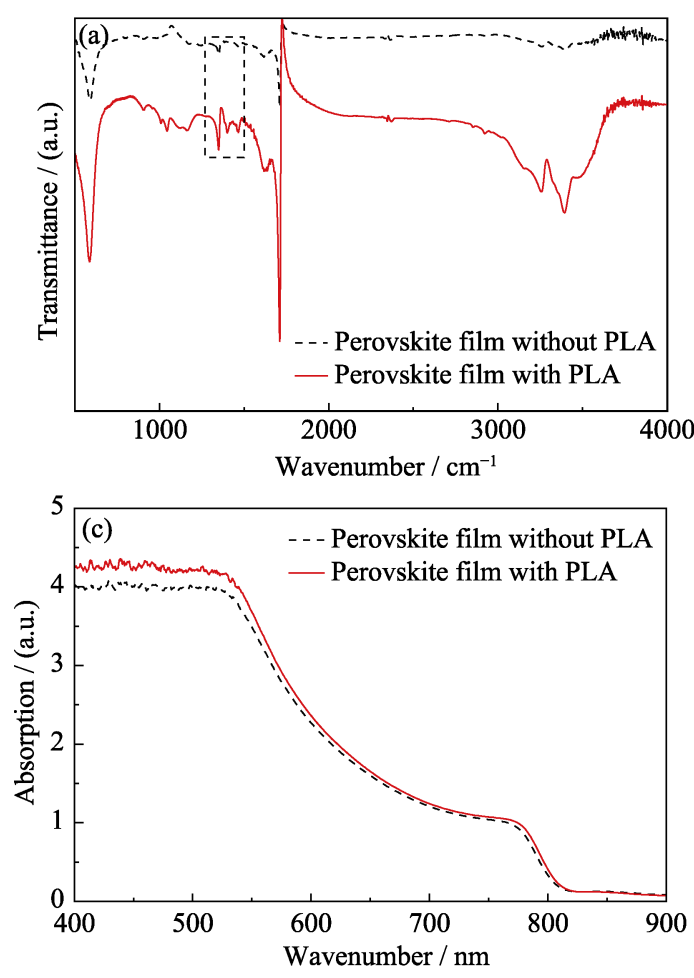

\subsection{PLA 修饰对钙钛矿太阳电池性能的影响}

为研究 PLA 分子对 PSC 光电性能的影响, 分别 制备了 30 个参照 PSC 和 PLA 修饰 PSC 并对其进行 $J-V$ 测试, 获得了光电性能参数的统计分布图(图 4) 和平均值及标准偏差(表 1)。从图表可知, PLA 修饰 器件的短路电流 $\left(J_{\mathrm{SC}}\right)$ 、开路电压 $\left(V_{\mathrm{OC}}\right)$ 和填充因子 $(F F)$ 均得到不同程度提升, 器件制作的可重复性良 好, 最佳 $\mathrm{PCE}$ 由 $20.10 \%$ 提高到 $21.53 \%$ 。 $J_{\mathrm{SC}}$ 的提升 可能是由于 PLA 溶液冲洗后 $\mathrm{PbI}_{2}$ 薄膜与有机盐反 应更充分 (图 3(d))、钙铁矿薄膜的光吸收性能增强 (图 3(c)) 的缘故。而钙钛矿薄膜内缺陷的针化则有利

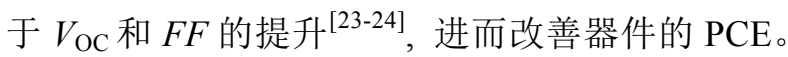

比较两种最优器件的 $J-V$ 曲线(图 $5(\mathrm{a} \sim \mathrm{c})$ ), 由 于 PSC 通常存在迟滞效应, 从低电压向高电压扫 描(正向扫描)与从高电压向低电压扫描(反向扫描) 获得的 $J-V$ 曲线并不重合, 为更准确地评价 PSC 性 能并计算器件的迟滞因子 ${ }^{[13]}$, 故对两个扫描方向的 $J-V$ 曲线均进行了测量。结果显示 PLA 修饰器件的 $J_{\mathrm{SC}}$ 和 $V_{\mathrm{OC}}$ 与参照器件接近, $\mathrm{PCE}$ 的增长主要来源于 $F F$ 的提升。 $F F$ 的影响因素较多, 而缺陷钝化是提 升 $F F$ 的一个重要因素 ${ }^{[25]}$ 。由于 PLA 修饰钻钛矿薄 膜晶界处富集 $\mathrm{PbI}_{2}($ 图 2(a,b)) 可延长载流子寿命, 降 低卤素空位浓度 ${ }^{[15]}$, 且 PLA 分子可与 $\mathrm{TiO}_{2}$ 表面的 带电缺陷和钙铁矿薄膜晶界处未配位有机阳离子
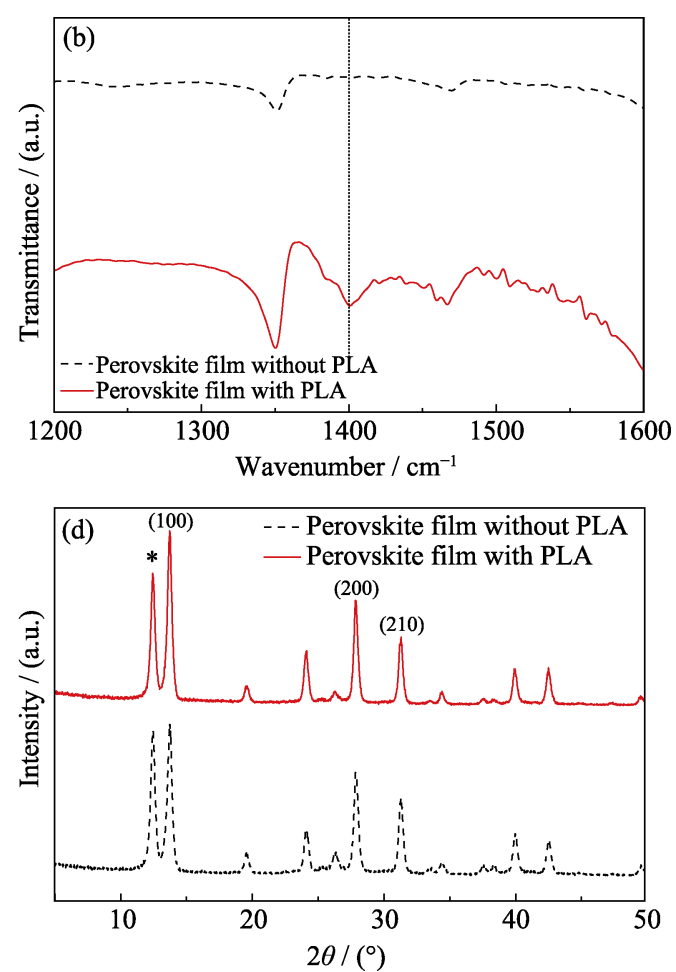

图 3 (a)钻钛矿薄膜的 ATR-FT-IR 光谱((b)为(a)中黑色虚线矩形区域的放大图), (c)薄膜的紫外-可见吸收光谱图和(d)XRD 图谱 Fig. 3 (a) ATR-FT-IR spectra, ((b) magnified ATR-FT-LR spectra of rectangular area in (a)), (c) UV-Vis absorption spectra and (d) XRD patterns of $\mathrm{FTO} / \mathrm{TiO}_{2} /$ perovskite films with and without PLA 

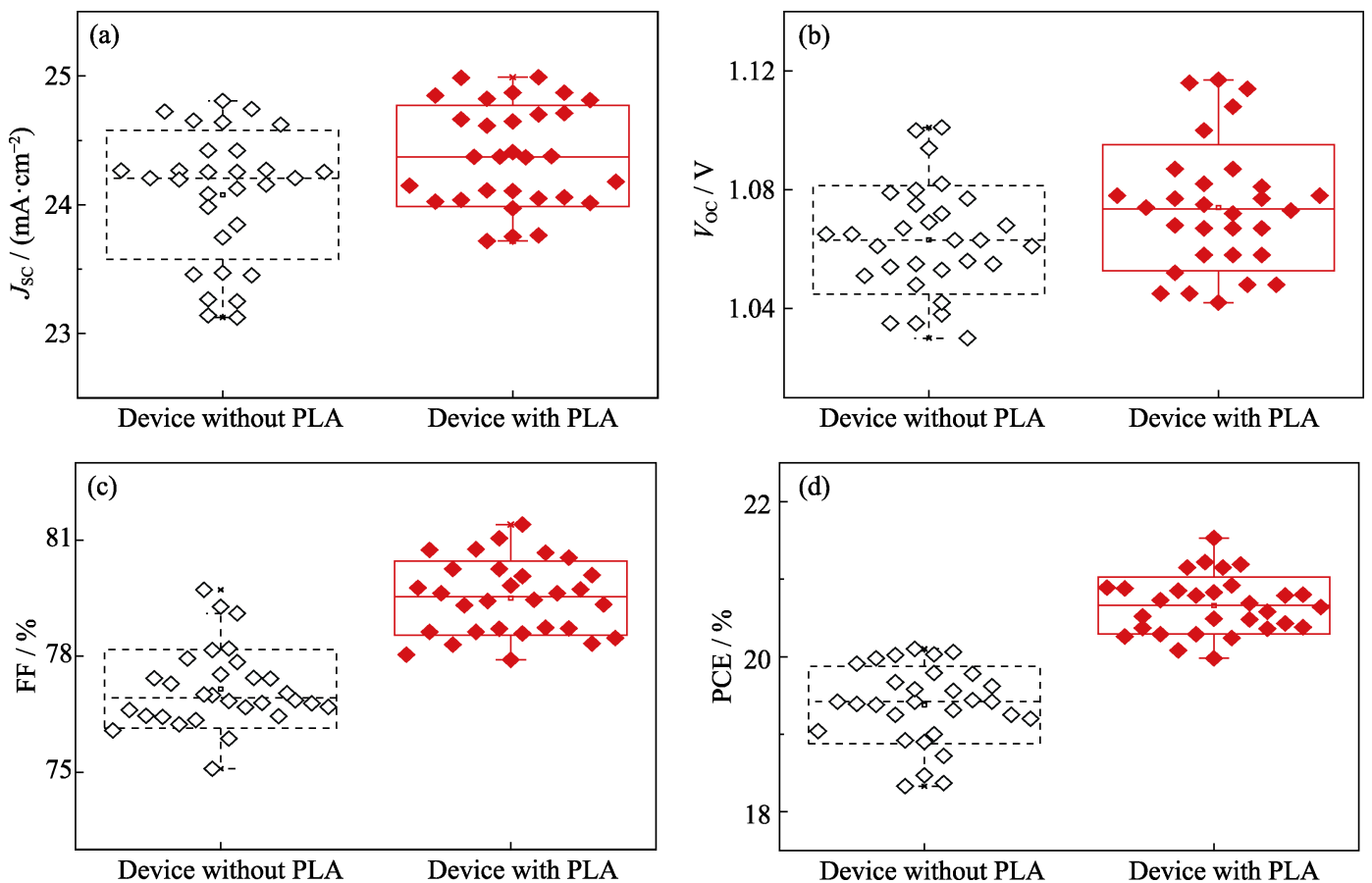

图 4 PLA 修饰及参照 $\mathrm{PSC}$ 器件的(a) $J_{\mathrm{SC}} 、(\mathrm{~b}) V_{\mathrm{OC}} 、(\mathrm{c}) F F$ 和(d)PCE 的统计分布图

Fig. 4 Statistical deviations of (a) $J_{\mathrm{SC}},(\mathrm{b}) V_{\mathrm{OC}},(\mathrm{c}) F F$, and (d) PCE for PSC devices without and with PLA

表 1 PLA 修饰及参照 PSC 器件光电性能参数的平均值和标准偏差

Table 1 Mean values and standard deviation (SD) of photovoltaic parameters for PSC devices without and with PLA

\begin{tabular}{ccccc}
\hline Device & $J_{\mathrm{SC}}(\mathrm{SD}) /\left(\mathrm{mA} \cdot \mathrm{cm}^{-2}\right)$ & $V_{\mathrm{OC}}(\mathrm{SD}) / \mathrm{V}$ & $F F(\mathrm{SD}) / \%$ & $\mathrm{PCE}(\mathrm{SD}) / \%$ \\
\hline Without PLA & $23.84(0.74)$ & $1.07(0.02)$ & $76.80(1.15)$ & $19.49(0.48)$ \\
With PLA & $24.37(0.50)$ & $1.07(0.02)$ & $78.74(1.43)$ & $20.55(0.39)$ \\
\hline
\end{tabular}
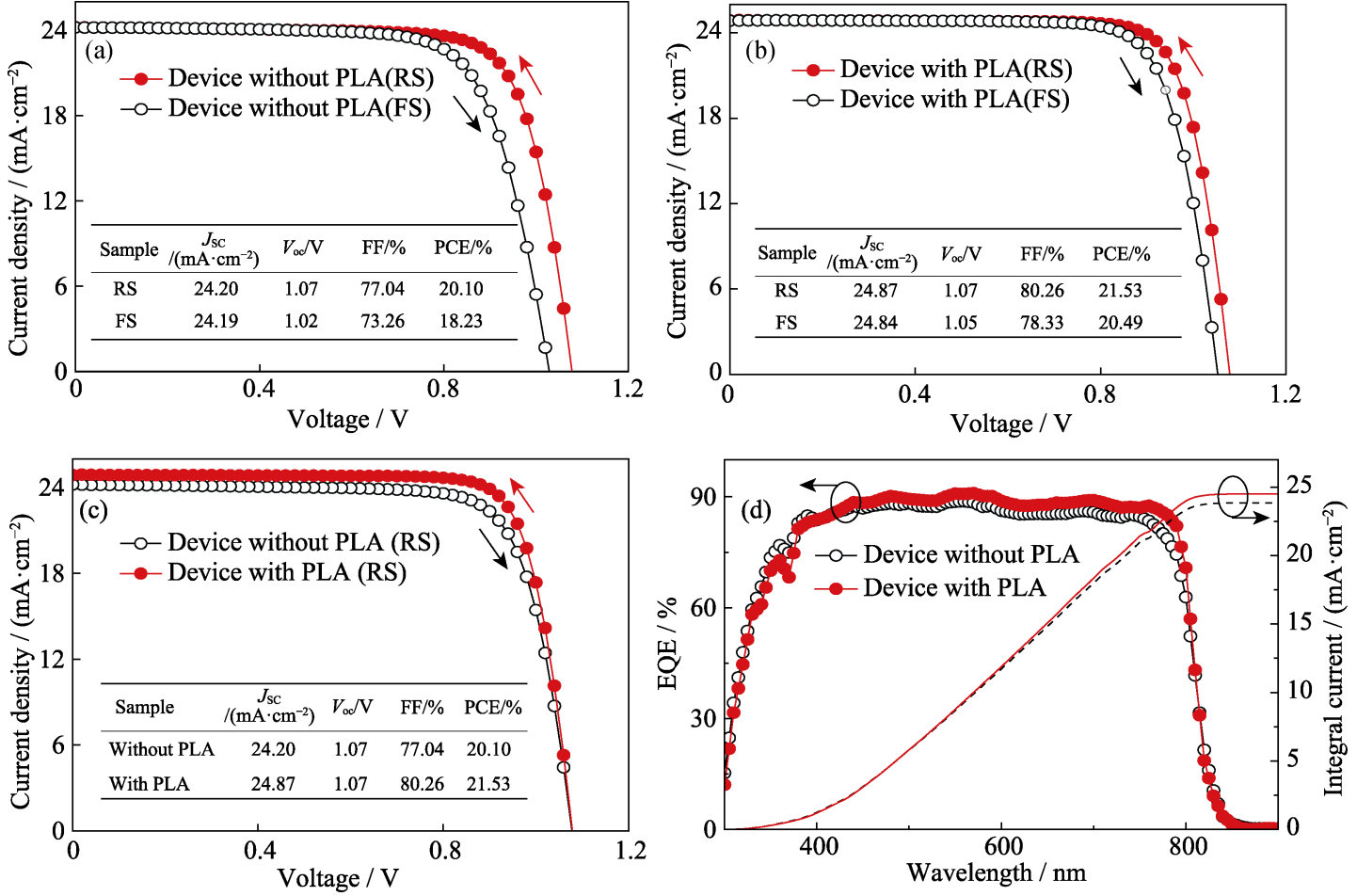

图 5 最优(a)参照和(b)PLA 修饰 PSC 器件的正反扫 $J-V$ 曲线, (c)反扫 $J-V$ 曲线的对比, (d)外量子效率谱图及 $J_{\mathrm{SC}}$ 积分曲线

Fig. 5 Forward and reverse scan $J$ - $V$ curves of the optimal PSC devices (a) without and (b) with PLA, (c) comparison of the reverse $J-V$ curves, and (d) external quantum efficiency spectra and integrated $J_{\mathrm{SC}}$ curves for the optimal PSC devices without and with PLA 
结合 ${ }^{[8]}$, 从而提升 PLA 修饰器件的 $F F$ 。除了 PCE 的提升, PLA 修饰器件的迟滞明显小于参照器件(图 $5(\mathrm{a}, \mathrm{b}))$, 两者的迟滞因子 ${ }^{[13]}$ 分别为 4.83\%和 9.30\%, 说明引入 PLA 对 $J-V$ 曲线的迟滞具有抑制作用, 这 可能与上述缺陷针化和载流子界面抽取的改善有 关。为验证器件 $J-V$ 结果的准确性, 对最优器件进行 $\mathrm{EQE}$ 测试, 图谱显示外量子效率在 400 800 nm 波长 范围内达到了 $70 \%$ 以上, 而最大值出现在 $550 \mathrm{~nm}$ 附 近(图 5(d)), 该波长范围内光子对电流具有显著贡 献。在上述波长范围内, PLA 修饰器件的 EQE 值略 高于参照器件, 这与两者的 $J_{\mathrm{SC}}$ 测量值相对应。通过 对 $\mathrm{EQE}$ 数值和 $\mathrm{AM} 1.5 \mathrm{G}$ 下光子通量 (Photon flux) 的乘积在 300 900 nm 波长范围内进行积分, 获得两 个器件的 $J_{\mathrm{SC}}$ 计算值(分别为 23.89 和 $24.54 \mathrm{~mA} \cdot \mathrm{cm}^{-2}$ ), 与图 5(c)对应的 $J_{\mathrm{SC}}$ 测量值几乎完全一致。

\subsection{PLA 修饰影响钙钛矿太阳电池的机理}

为进一步探究 PLA 分子对钙钛矿薄膜缺陷的 钝化作用, 对不含电荷传输层的钙钛矿薄膜(玻璃) 钙钛矿薄膜) 进行稳态 PL 和 TRPL 表征。稳态 PL 表征显示两样品的苂光发射峰位置一致, 表明 PLA 的引入对钲钛矿薄膜的晶体结构无明显影响, 这与 XRD 的结果(图 3(d))一致。然而, PLA 修饰样品的 荧光强度明显强于参照样品(图 6(a))。由于样品不具 备电荷传输层, 光生载流子无法通过电荷抽取而促 使苂光湮灭, 因此苂光强度增强表明钙钛矿薄膜陷 阱态密度降低 ${ }^{[26]}$ 。

TRPL 衰减图谱可用于揭示器件的载流子动力 学, 反映器件内部载流子寿命以及复合速率。本研 究测得的苂光强度衰减曲线采用时间 $t$ 的双指数函 数进行拟合, 拟合公式(1)如下:

$$
I(t)=A_{1} \exp \left(-t / \tau_{1}\right)+A_{2} \exp \left(-t / \tau_{2}\right)
$$

其中, $t$ 为光激发时间, $I(t)$ 为 $t$ 时间下对应的苂光强 度, $\tau_{1}$ 对应材料表面性质, 为快速瞬态寿命, $\tau_{2}$ 对应 材料内部性质, 为慢衰减寿命, $A_{1}$ 和 $A_{2}$ 代表快速瞬 态寿命和慢衰减寿命的贡献系数。图 6(b)及其拟合 结果(c)显示 PLA 修饰的钙钛矿薄膜苂光衰减更慢, $\tau_{1} 、 \tau_{2}$ 和平均寿命 $\left(\tau_{\mathrm{ay}}\right)$ 更长 ${ }^{[27]}$ 。由于样品的祄底为玻 璃, 不含电荷传输层, 钙钛矿薄膜的荧光衰减与载 流子的非辐射复合密切相关, 苂光寿命较长说明非 辐射复合被抑制, 导致 PLA 修饰样品的陷阱态密度 降低, 有助于器件 $F F$ 的提升。

为定量表征 PLA 添加剂对钙钛矿薄膜陷阱态 密度的影响, 本课题组制备了纯电子注入器件, 其 结构为 $\mathrm{FTO} / \mathrm{TiO}_{2} /$ 钙铁矿/ $\mathrm{PCBM} / \mathrm{Au}$ (图 7(a)), 采用 SCLC 对器件进行测试 ${ }^{28]}$ 。器件测得的 $I-V$ 曲线含
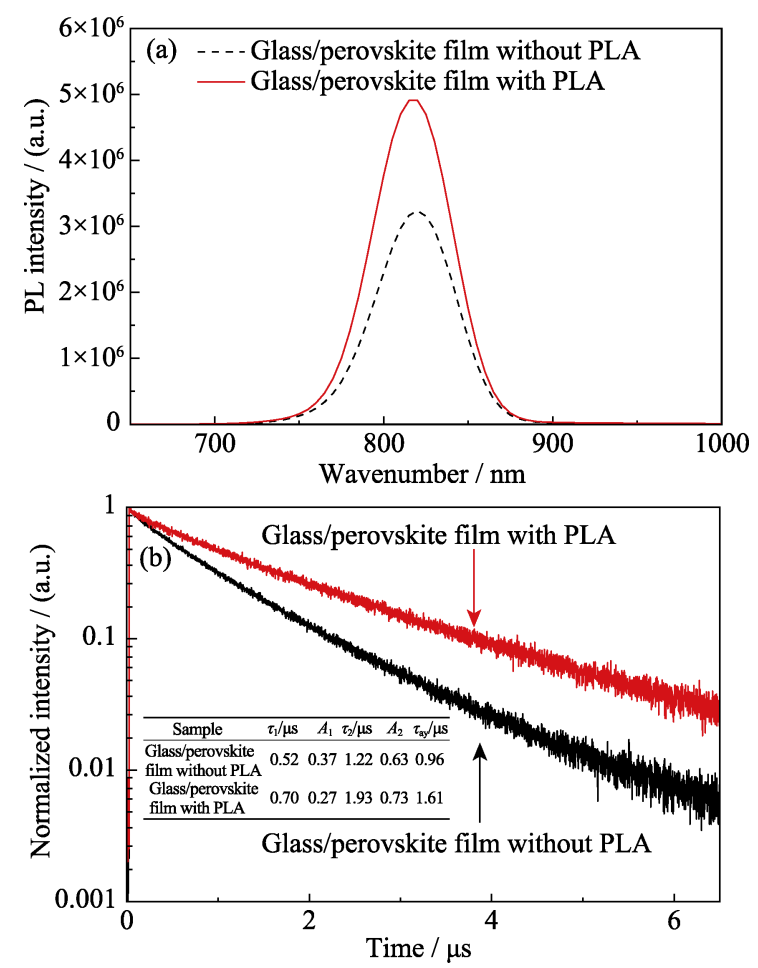

图 6 PLA 修饰及参照玻璃/钻钛矿薄膜的(a)稳态 PL 光谱, (b)TRPL 衰减图谱 $\left(\lambda_{\text {excitation }}=510 \mathrm{~nm}\right)$ 和 TRPL 的拟合结果 Fig. 6 (a) PL spectra, (b) TRPL spectra $\left(\lambda_{\text {excitation }}=510 \mathrm{~nm}\right)$ and TRPL fitting results of glass/perovskite films with and without PLA

有三个不同的区域，分别为欧姆区域、子区域和陷 阱填充限制区域(TFL), 分别用红色、绿色和黄色线 段表示(图 7(b))。利用欧姆区到 TFL 区转折点处对 应的电压值 $\left(V_{\mathrm{TFL}}\right)$ 和公式(2)可计算接近价带顶的陷 阱态密度 ${ }^{[29]}$ :

$$
V_{\mathrm{TFL}}=e n_{\mathrm{t}} L^{2} / 2 \varepsilon \varepsilon_{0}
$$

其中, $e=1.602 \times 10^{-19} \mathrm{C}, n_{\mathrm{t}}$ 是陷阱态密度, $L$ 为两电极 间的晶体厚度, $\varepsilon$ 是钻钛矿的相对介电常数, 取 $\mathrm{FAPbI}_{3}$ 的相对介电常数(46.9)作为近似值, $\varepsilon_{0}$ 是真空 介电常数 $\left(8.8542 \times 10^{-14} \mathrm{~F} \cdot \mathrm{cm}^{-1}\right)$ 。图 7(b) 显示参照样 品和 PLA 修饰样品的 $V_{\mathrm{TFL}}$ 分别为 0.69 和 $0.42 \mathrm{~V}$, 计 算得到 $n_{\mathrm{t}}$ 分别为 $5.59 \times 10^{16}$ 和 $3.40 \times 10^{16} \mathrm{~cm}^{-3}$, 表明 PLA 修饰后, 钙钛矿薄膜的电子陷阱态密度降低。 该结果直接证明了引入 PLA 分子可钝化钙钛矿薄 膜的缺陷。

图 S2 (a, b) 为 PSC 的 EIS 阻抗谱图, 数据点分别 为器件在光照和暗态条件下测得的实际阻抗值, 实 线为按图 S2(a)插图中等效电路进行拟合的结果, 均呈现 2 个半圆弧状, 其中左半圆弧位于高频区域, 代表界面电荷传输阻抗 $R_{1}$, 反映界面上电荷转移的 难易程度; 右半圆弧位于低频区域, 为电荷复合阻 抗 $R_{2}$, 反映电荷复合受到抑制的程度; 左半圆弧高 频段在实轴上的截距代表器件的串联电阻 $R_{\mathrm{S}}{ }^{[30-32]}$ 。 

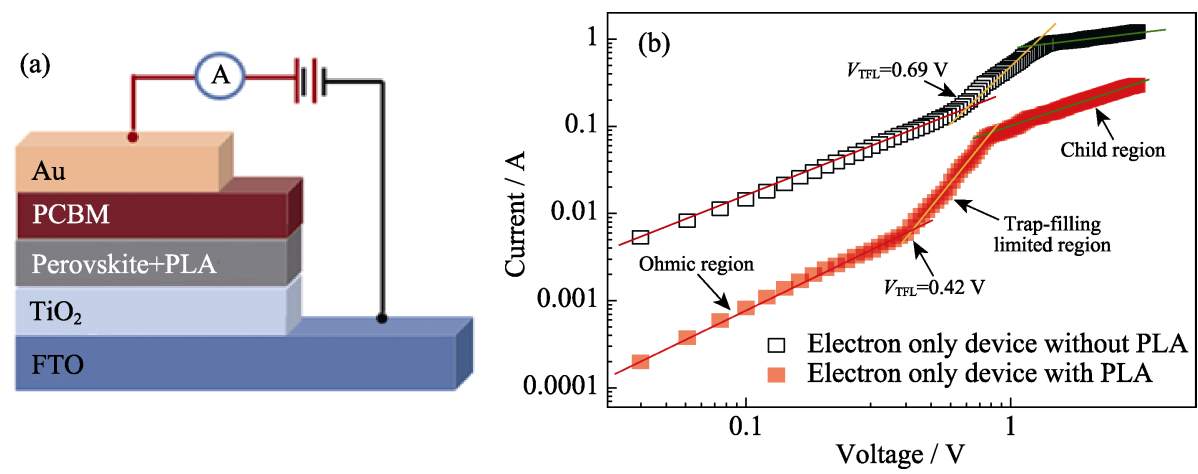

图 7 (a)PLA 修饰钲钛矿薄膜纯电子注入器件的结构示意图, (b)PLA 修饰及参照钙钛矿 薄膜纯电子注入器件的 SCLC 测试结果

Fig. 7 (a) Schematic diagram of electron-only perovskite film device with PLA, and (b) SCLC measurements of electron-only perovskite film devices with and without PLA

$R_{1} 、 R_{2}$ 和 $R_{\mathrm{S}}$ 的具体数值通过拟合获得, 归纳在表 $\mathrm{S} 1$ 中。 $V_{\mathrm{OC}}$ 主要与光吸收层的半导体材料带隙、器件 各层的能级匹配、载流子的输运与复合有关, 载流 子输运改善、复合减少, 有利于 $V_{\mathrm{OC}}$ 的提高。 $F F$ 与 光伏器件等效电路的串联电阻和并联电阻有关, 其 中并联电阻又与载流子的复合有关, 较小的串联电 阻和较大的并联电阻有助于获得较高的 $F F$ 。 $R_{1}$ 减 小优化了载流子输运, 界面处载流子的抽取得到改 善。 $R_{2}$ 增大表明载流子复合受到抑制, 器件的并联 电阻增大。因此, 基于上述分析, 在半导体材料带隙 和器件各层能级不变的前提下, PLA 修饰 PSC 的 $R_{1}$ 减小、 $R_{2}$ 增大、 $R_{\mathrm{S}}$ 不变, 有助于提高器件的 $V_{\mathrm{OC}}$ 和 $F F$ 。 又由于 $J_{\mathrm{SC}}$ 主要与吸收的光子数量有关 ${ }^{[33-34]}$, 在其基 本不变的前提下, $V_{\mathrm{OC}}$ 和 $F F$ 的提升有助于改善 $\mathrm{PCE}$ 。 结合 TRPL 和 SCLC 表征的相关结果, 表明 PLA 修 饰不仅可以通过降低陷阱态密度抑制电子和空穴的 复合, 还可改善载流子在界面处的输运, 因而器件 的 $V_{\mathrm{OC}} 、 F F$ 和 $\mathrm{PCE}$ 均得到了明显提高(图 5) ${ }^{[35]}$ 。

\section{3 结论}

本文通过两步溶液法制备了器件结构为 $\mathrm{FTO} / \mathrm{TiO}_{2} /$ perovskite/ $\mathrm{HTL} / \mathrm{Au}$ 的钙钛矿太阳电池, 并在钙钛矿薄膜内部引入有机小分子 PLA。研究结 果显示该添加剂可以改善钙钛矿薄膜的光吸收性能, 将钙钛矿载流子的平均寿命从 $0.96 \mu \mathrm{s}$ 延长至 $1.61 \mu \mathrm{s}$ ，材料的陷阱态密度从 $5.59 \times 10^{16} \mathrm{~cm}^{-3}$ 降低到 $3.40 \times 10^{16} \mathrm{~cm}^{-3}$, 并改善载流子输运, 从而提高器件 的 $V_{\mathrm{OC}} 、 F F$ 和 PCE。PLA 修饰 PSC 的最佳 PCE 达 到 $21.53 \%$, 较参照器件 $(20.10 \%)$ 有明显提高。这可 能与 PLA 导致的晶界处 $\mathrm{PbI}_{2}$ 富集和 PLA 分子在 $\mathrm{TiO}_{2}$ 表面和钙铁矿晶界处的针定有关。本研究可以 为针化钙钛矿薄膜界面缺陷提供借鉴。

\section{补充材料}

本文相关补充材料可登陆 https://doi.org/10.15541/ jim20200495 查看。

\section{参考文献:}

[1] KOJIMA A, TESHIMA K, SHIRAI Y, et al. Organometal halide perovskites as visible-light sensitizers for photovoltaic cells. J. Am. Chem. Soc., 2009, 131(17): 6050-6051.

[2] Best Research-Cell Efficiency Chart. https://www.nrel.gov/pv/cellefficiency.html.

[3] GREEN M A. The path to $25 \%$ silicon solar cell efficiency: history of silicon cell evolution. Prog. Photovolt: Res. Appl., 2009, 17: 183-189.

[4] XIONG H, ZHANG B X, JIA W, et al. Polymer PVP additive for improving stability of perovskite solar cells. J. Inorg. Mater., 2019, 34(1): 96-102.

[5] YU S W, ZHAO Z W, ZHAO J J, et al. Research progress in novel in-situ integrative photovoltaic-storage tandem cells. J. Inorg. Mater., 2020, 35(6): 623-632.

[6] CHU Z Y, LI G L, JIANG Z H, et al. Recent progress in high-quality perovskite $\mathrm{CH}_{3} \mathrm{NH}_{3} \mathrm{PbI}_{3}$ single crystal. J. Inorg. Mater, 2018, 33(10): 1035-1045.

[7] PATIL J V, MALI S S, HONG C K. A thiourea additive-based quadruple cation lead halide perovskite with an ultra-large grain size for efficient perovskite solar cells. Nanoscale, 2019, 11: 21824-21833.

[8] ZHANG S, LU Y T, LIN B C, et al. PVDF-HFP additive for visible-light-semitransparent perovskite films yielding enhanced photovoltaic performance. Sol. Energy Mat. Sol. C, 2017, 170: $178-186$.

[9] ZHENG X P, CHEN B, DAI J, et al. Defect passivation in hybrid perovskite solar cells using quaternary ammonium halide anions and cations. Nat. Energy, 2017, 2: 17102.

[10] DE ROO J, IBÁÑEZ M, GEIREGAT P, et al. Highly dynamic ligand binding and light absorption coefficient of cesium lead bromide perovskite nanocrystals. ACS Nano, 2016, 10: 2071-2081.

[11] YANG S, DAI J, YU Z H, et al. Tailoring passivation molecular structures for extremely small open circuit voltage loss in perovskite solar cells. J. Am. Chem. Soc., 2019, 141(14): 5781-5787. 
[12] BARLY I L, DEQUILETTES D W, PAZOS-QUTON, et al. Hybrid perovskite films approaching the radiative limit with over $90 \%$ photoluminescence quantum efficiency. Nat. Photonics, 2018, 12: 355-361.

[13] YANG J, LIU C, CAI C, et al. High performance perovskite solar cells with excellent humidity and thermo stability via fluorinated perylenediimide. Adv. Energy. Mater, 2019, 9(18): 1900198.

[14] ZHANG S, HU Z L, ZHANG J, et al. Interface engineering via phthalocyanine decoration of perovskite solar cells with high efficiency and stability. J. Power Sources, 2019, 438: 226987.

[15] WANG H H, WANG Z W, YANG Z, et al. Ligand-modulated excess $\mathrm{PbI}_{2}$ nanosheets for highly efficient and stable perovskite solar cells. Adv. Mater., 2020, 32(21): 2000865.

[16] NIU T Q, LU J, MUNIR R, et al. Stable high-performance perovskite solar cells via grain boundary passivation. Adv. Mater, 2018, 30(16): 1706576

[17] DE WOLF S, HOLOVSKY J, MOON S J, et al. Organometallic halide perovskites: sharp optical absorption edge and its relation to photovoltaic performance. J. Phys. Chem. Lett., 2014, 5(6): 1035-1039.

[18] STRANKS S D, EPERON G E, GRANCINI G, et al. Electron-hole diffusion lengths exceeding 1 micrometer in an organometal trihalide perovskite absorber. Science, 2013, 342(6156): 341-344.

[19] CHEN J H, ZUO L J, ZHANG Y Z, et al. High-performance thickness insensitive perovskite solar cells with enhanced moisture stability. Adv. Energy Mater, 2018, 8(23): 1800438.

[20] WHEELER L M, SANEHIRA E M, MARSHALL A R, et al. Targeted ligand-exchange chemistry on cesium lead halide perovskite quantum dots for high-efficiency photovoltaics. J. Am. Chem. Soc., 2018, 140(33): 10504-10513.

[21] IM J H, JANG I H, PELLET N, et al. Growth of $\mathrm{CH}_{3} \mathrm{NH}_{3} \mathrm{PbI}_{3}$ cuboids with controlled size for high-efficiency perovskite solar cells. Nat. Nanotechnol., 2014, 9(181): 927-932.

[22] LIU Y, MONOJIT B, LAWRENCE A R, et al. Understanding interface engineering for high-performance fullerene/perovskite planar heterojunction solar cells. Adv. Energy Mater, 2015, 6: 1501606.

[23] WU Y H, WANG P, ZHANG W H. Heterojunction engineering for high efficiency cesium formamidinium double-cation lead halide perovskite solar cells. ChemSusChem, 2018, 11(5): 837-842.

[24] HOU Y, DU X Y, SIMON S, et al. A generic interface to reduce the efficiency-stability-cost gap of perovskite solar cells. Science, 2017, 358(6367): 1192-1197.

[25] ZHANG J, SUN Q, CHEN Q Y, et al. High efficiency planar p-i-n perovskite solar cells using low-cost fluorene-based hole transporting material. Adv. Funct. Mater., 2019, 29: 1900484.

[26] CHEN H Y, ZHAN Y, XU G Y, et al. Organic n-type molecule: managing the electronic states of bulk perovskite for high-performance photovoltaics. Adv. Funct. Mater, 2020, 120(6): 8267-8302.

[27] DEQUILETTES D W, VORPAHL S M, STRANKS S D, et al. Impact of microstructure on local carrier lifetime in perovskite solar cells. Science, 2015, 348(6235): 683-686.

[28] WANG L, LIU H, LIU C W, et al. Approaching optimal hole transport layers by organic monomolecular strategy for efficient inverted perovskite solar cells. J. Mater. Chem. A, 2020, 4(6): 1-21.

[29] HOU M N, XU Y Z, ZHOU B, et al. Aryl diammonium iodide passivation for efficient and stable hybrid organ-inorganic perovskite solar cells. Adv. Funct. Mater, 2020, 30(34): 2002366.

[30] SON D Y, KIM S G, SEO J Y, et al. Universal approach toward hysteresis free perovskite solar cell via defect engineering. J. Am. Chem. Soc., 2018, 140(4): 1358-1364.

[31] GUERRERO A, GARCIA-BELMONTE, MORA-SERO I, et al. Properties of contact and bulk impedances in hybrid lead halide perovskite solar cells including inductive loop elements. J. Phys. Chem. C, 2016, 120(15): 8023-8032.

[32] WANG Q, MOSER J E, GRTZEL M. Electrochemical impedance spectroscopic analysis of dye-sensitized solar cells. J. Phys. Chem. $B, 2005$, 109(31): 14945-53.

[33] ZHANG S, DONG G Y, LIN B C, et al. A polymer gel electrolyte with an inverse opal structure and its effects on the performance of quasi-solid-state dye-sensitized solar cells. J. Power Sources, 2015, 277: 52-58.

[34] ZHANG S, DONG G Y, LIN B C, et al. Performance enhancement of aqueous dye-sensitized solar cells via introduction of a quasisolid-state electrolyte with an inverse opal structure. Sol Energy, 2016, 127: 19-27.

[35] YANG J A, XIAO A D, XIE L S, et al. Precise control of $\mathrm{PbI}_{2}$ excess into grain boundary for efficacious charge extraction in off-stoichiometric perovskite solar cells. Electrochim. Acta, 2020, 338: 135697. 


\section{补充材料:}

\section{L-3-(4-吡啶基)-丙氨酸钝化钙钛矿太阳电池界面缺陷}

刘雯雯 ${ }^{1}$, 胡志蕾 ${ }^{1}$, 王 立 $^{1}$, 曹梦莎 ${ }^{1}$, 张 晶 $^{1}$, 张 婧 ${ }^{1}$, 张 帅 ${ }^{1}$, 袁宁一 1 , 丁建宁 ${ }^{2}$

( 1. 常州大学, 江苏省光伏科学与工程协同创新中心 材料科学与工程学院, 常州 $213164 ; 2$. 江苏大学微纳科学与 技术中心，镇江 212013)
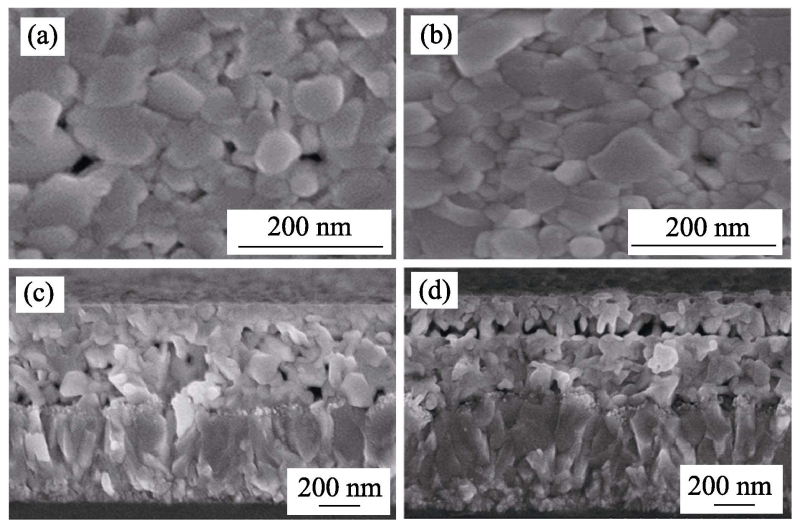

图 S1 (a, c) 参照和 $(b, d) P L A$ 修饰 $\mathrm{FTO} / \mathrm{TiO}_{2} / \mathrm{PbI}_{2}$ 薄膜的(a,b)表面和 $(\mathrm{c}, \mathrm{d})$ 截面 SEM 照片

Fig. S1 (a,b) Surface and (c,d) cross sectional SEM images of FTO/TiO $/ \mathrm{PbI}_{2}$ film without PLA (a, c) and with PLA (b, d)
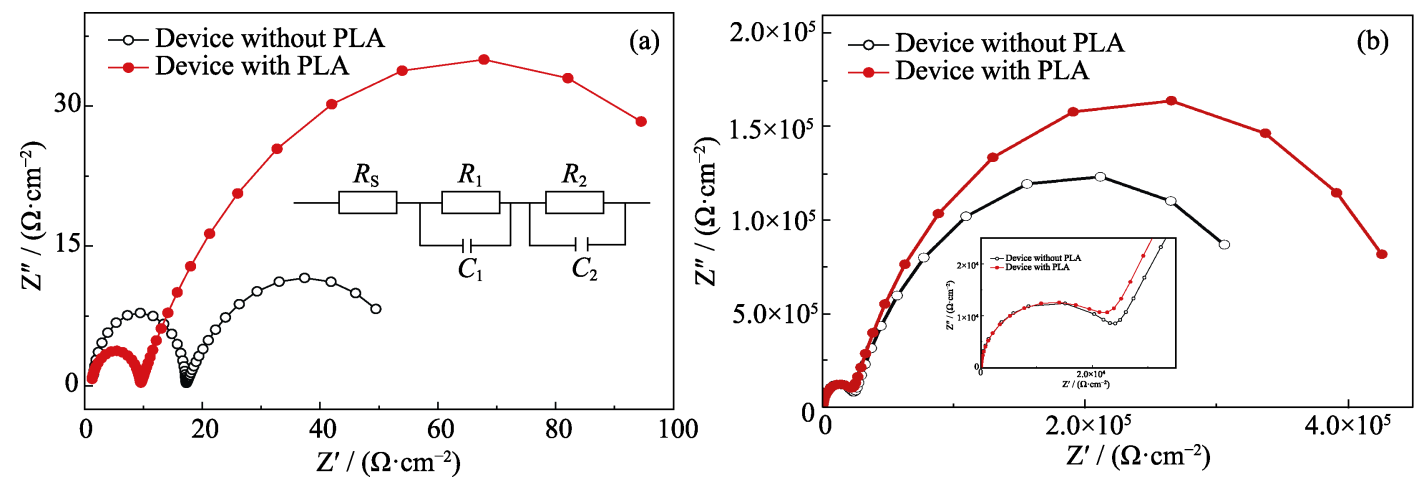

图 S2 (a)光照和(b)暗态环境下参照和 PLA 修饰 PSC 的 Nyquist 图谱

Fig. S2 EIS plots of PSC without and with PLA (a) under illumination and (b) in dark

表 S1 光照和暗态环境下参照及 PLA 修饰 PSC 器件的 EIS 拟合参数

Table S1 EIS fitting parameters of PSC without and with PLA under illumination and in dark

\begin{tabular}{cccc}
\hline Device & $R_{\mathrm{S}} /\left(\Omega \cdot \mathrm{cm}^{-2}\right)$ & $R_{1} /\left(\Omega \cdot \mathrm{cm}^{-2}\right)$ & $R_{2} /\left(\Omega \cdot \mathrm{cm}^{-2}\right)$ \\
\hline Without PLA (Illumination) & 1.80 & 58.70 & 7.90 \\
With PLA (Illumination) & 1.43 & 15.74 & 40.49 \\
Without PLA (Dark) & 0.78 & $3.46 \times 10^{5}$ & $2.27 \times 10^{4}$ \\
With PLA (Dark) & 1.21 & $2.15 \times 10^{4}$ & $4.50 \times 10^{5}$ \\
\hline
\end{tabular}

